\title{
Sporadic Creutzfeldt-Jakob disease: a case report of long disease duration and difficulties in confirming the diagnosis with short literature review
}

\author{
Anna Karolina Stanowska ${ }^{1}$, Barbara Wach ${ }^{1}$, Izabela Herman-Sucharska², Dariusz Adamek ${ }^{3}$ \\ ${ }^{1}$ Department of Neurology, $5^{\text {th }}$ Military Hospital with Polyclinic, Cracow, Poland, ${ }^{2}$ Department of Radiology, Faculty of Health \\ Sciences, Jagiellonian University, Medical College, Cracow, Poland, ${ }^{3}$ Department of Neuropathology, Institute of Neurology, \\ Jagiellonian University, Medical College, Cracow, Poland
}

\begin{abstract}
Creutzfeldt-Jakob disease (CID) is a spongiform encephalopathy with the fatal outcome, caused by the accumulation of pathological prion protein in the central nervous system (CNS). CJD is classified into four types: sporadic (SCJD), familial or genetic (fCJD), iatrogenic (iCJD) and variant form (VCJD). The recognition of CJD is based on the clinical presentation, neuroimaging, electroencephalography and biochemical tests. The hyperintense signals in basal ganglia on brain magnetic resonance imaging (MRI), periodic sharp and slow wave complexes (PSWCS) in the electroencephalogram as well as presence of neuronal proteins such as protein 14-3-3 in the cerebrospinal fluid (CSF) support the diagnosis. The definite diagnosis of CJD still demands neuropathological confirmation.

We report the case of a 56-year-old woman with the rapidly progressive cognitive impairment, motor dysfunctions and the fulminant neurological deterioration to akinetic mutism during the five weeks' hospitalisation. The probable diagnosis of SCID was based on medical history and characteristic findings in MRI. The positive result of the real-time quaking-induced conversion (RT-QuIC) test and presence of protein 14-3-3 were obtained post-mortem and definite diagnosis was confirmed by neuropathological examination.

In this paper we would like to emphasize the difficulties in reaching the diagnosis and the need for a series of diagnostic examinations in different points of time to obtain the confirming results.
\end{abstract}

Key words: Creutzfeldt-Jakob disease, CJD phenotypes, prion disease, dementia.

\section{Case presentation}

A 56-year-old woman, a butcher worker, was admitted to the neurology ward after transient loss of consciousness with a history of progressive cognitive impairment and motor dysfunctions. The initial symptoms including behavioural changes started about one year before the admission. She was peri- odically hyperactive and behaved inappropriately to the situation. The patient's family also observed some motor symptoms like: involuntary movements of her upper limbs (later diagnosed as myoclonic movements), falling out of objects from her hands as well as gait unsteadiness causing falls. The psychomotor retardation occurred, her speech became dys- 
arthric. She became apathetic and withdrew from the social life.

She was complaining of headaches, the neck pain, paraesthesia in hands (numbness and tingling) and fluctuations of arterial blood pressure. During the first year of symptoms she underwent the magnetic resonance imaging (MRI) of the brain and the cervical spine. The brain MRI revealed meningioma of the right fronto-parietal region, which was qualified for Gamma Knife radiosurgery. The results of cervical spine MRI showed C5/C6 and C6/C7 disc herniation without myelopathy, which was also intended to be treated surgically. The significant deterioration of her neurological condition occurred about 3-4 weeks before the hospitalization. Because of the observed dystonic movements of the upper limbs the operations were postponed. In her past medical history there was an episode of bilateral peroneal nerve palsy four years earlier, supposedly related to the work in a squat position.

On admission to the ward the patient was conscious, her autopsychic and allopsychic orientation in brief mental status examination was within basic level. Her general somatic state, including circulatory and respiratory functions, were within normal range. The neurological examination revealed four-limb rigidity with symmetrical hyperreflexia and dystonic movements of her upper limbs, accentuated more on the right side, the superficial sensory disturbances on the left side and the deep sensory disturbances in lower extremities. The patient was unable to move independently and was wheelchair-bound. She was complaining of poor eyesight and unspecific paraesthesia. The neuropsychological examination, which was only feasible to conduct in the initial phase of her hospitalization, revealed unequivocal deterioration of cognitive functions (slowing-down of thoughts and speech, delayed response to verbal tasks and memory impairment). The disturbances of superficial and deep sensory were manifested in variable fashion in the following days. Later on her visual problem severely aggravated, she developed cerebellar symptoms (four limbs ataxia), extrapyramidal dysfunctions (asymmetric rigidity of four extremities, more in the upper extremities and on the left side), hyperreflexia, and subtle left side hemiplegia with Babinski sign. During the four weeks of hospitalization her general conditions worsened significantly. The rapid deterioration of cognitive functions and impaired consciousness occurred. We observed myoclonus affecting upper extremities, aggravated dystonic movements of the trunk, upper and lower limbs as well as tonic epileptic seizures. Approximately two weeks before her death she developed akinetic mutism and passed away after six weeks of hospitalization.

The initial MRI brain examination was not unequivocal (Fig. 1A-C). It revealed hyperintensity in the region of the right caudate and the inner capsule on DWI with no contrast enhancement and no ischemic changes. These findings were suspected to be rather motor artefacts at first glance.

The laboratory tests, including blood tests, glucose levels, renal, hepatic and thyroid function tests, electrolytes, $C$-reactive protein (CRP), and $B_{12}$ vitamin levels were normal. Tests for human immunodeficiency virus (HIV), Treponema pallidum (IgM and $\operatorname{lgG}$ ), Borrelia (IgM and $\operatorname{lgG}$ ), anti-thyroid peroxidase, and anti-thyroglobulin were negative.

The results of cerebrospinal fluid were irrelevant (Table I). The polymerase chain reaction (PCR) assay of CSF for detection of encephalitis [E. coli, H. influenzae, L. monocytogenes, N. meningitidis, S. agalactiae, S. pneumoniae, cytomegalovirus (CMV), enterovirus, herpes simplex virus 1 (HSV 1), HSV 2, human herpesvirus 6 (HHV6), human parechovirus (HPeV), varicella zoster virus (VZV), and C. neoformans/gatti] was negative. The results of serum protein electrophoresis did not reveal any abnormalities. Apart from serum IgM-type anti-GM2 antibodies, no other paraneoplastic antibodies (anti-Hu, anti-Yo, anti-Ri, anti PNMA2, anti-CV2, anti-amphisin, anti-GM1, anti-GM3, anti-GD1a, antiGD1b, anti-GT1b, anti-GQ1b) were detected. Apart from the right peroneal nerve neuropathy there was no evidence of polyneuropathy in the electromyography. The serum-copper and the serum-ceruloplasmin levels as well as the urine-copper level were within normal range. Thus, the inflammation of the CNS and metabolic disorders were excluded. The test for protein 14-3-3 in the CSF, with the use of Western-Blot method, was negative. During hospitalisation the fluctuations of blood pressure and tachycardia were observed.

The EEG showed high-voltage theta and delta waves on the right hemisphere (the localisation of suspected meningioma) with periodic epileptic discharges. She was treated with antiepileptic drugs (diazepam, valproic acids, thiopental) and the consecutive EEG tests revealed progression towards diffused slow waves without epileptic discharges. Thus, the EEG pattern did not allow to recognise or to indicate any specific brain pathology.

No response to the intravenous steroid therapy and the course of plasmapheresis was observed. 


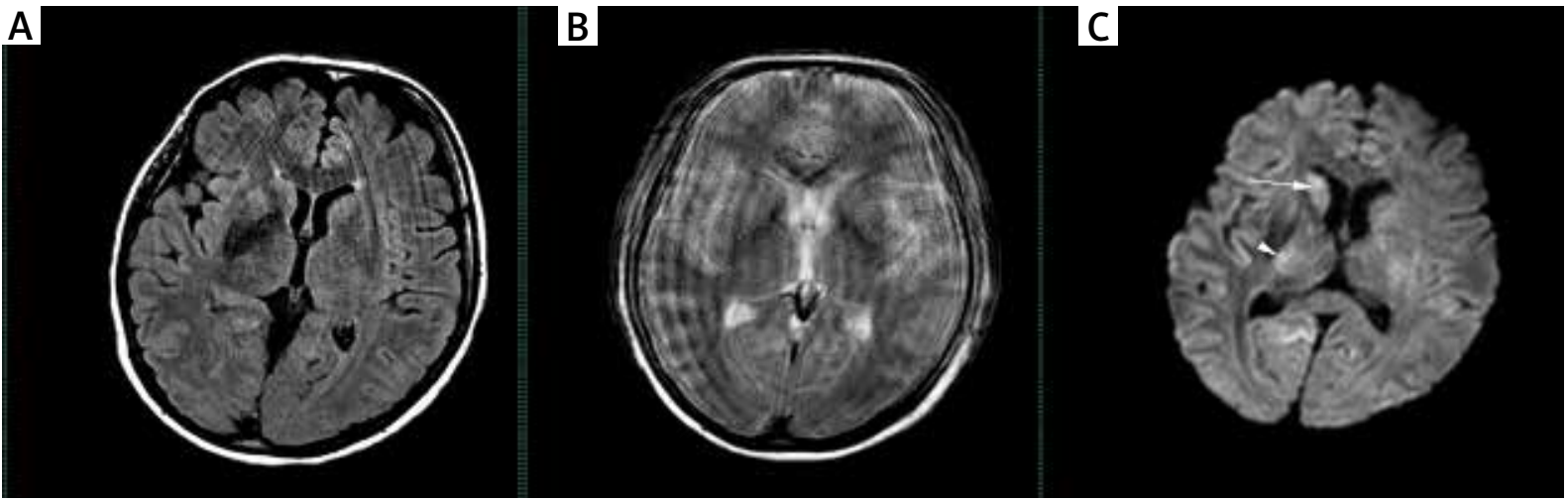

Fig. 1. Brain MRI, axial plane, sequences: A) FLAIR T2, B) TSET2, C) DWI SENSE: hyperintensity in the region of the right caudate (arrow) and the inner capsule (arrowhead) on DWI sequence.

Table I. Cerebrospinal fluid analysis

\begin{tabular}{|c|c|c|c|}
\hline Variable & 22 June & 28 June & 10 July \\
\hline Appearance & Clear & Clear & Clear \\
\hline Cytosis & $7 / 3$ & $12 / 3$ & $3 / 5$ \\
\hline Nonne-Apelt reaction & Negative & Poorly positive & Negative \\
\hline Pandy's test & Positive & Positive & Negative \\
\hline $\mathrm{Cl}$ CSF $[\mathrm{mmol} / \mathrm{l}]$ & 128 & 127 & 125 \\
\hline Glucose CSF $[\mathrm{mmol} / \mathrm{l}]$ & 4.8 & 4.5 & 4.5 \\
\hline $\begin{array}{l}\text { Lactate CSF } \\
(\mathrm{N}: 1.1-2.4 \mathrm{mmol} / \mathrm{l})\end{array}$ & 1.7 & 1.6 & 1.9 \\
\hline $\begin{array}{l}\text { Protein CSF } \\
(\mathrm{N}: 0.15-0.45 \mathrm{~g} / \mathrm{l})\end{array}$ & 0.49 & 0.53 & 0.6 \\
\hline
\end{tabular}

The results of the tests for the surface onconeural antigens (including anti-NMDAR, anti-AMPA, antiGABA B, anti-DPPX, anti-CASPR2, anti-LGI1) were negative.

In different stages of the disease in differential diagnosis we took into consideration the following conditions: the epileptic seizures due to meningio$\mathrm{ma}$, the posterior reversible encephalopathy syndrome and the autoimmunological encephalitis. Ultimately, considering the clinical picture and results of the tests, Creutzfeldt-Jakob disease has been considered especially since the alternative diagnoses had become rather improbable or excluded, particularly regarding the development of changes in MRI.

The brain MRI in the $4^{\text {th }}$ week hospitalisation showed the symmetrical hyperintensity of the basal ganglia (caudate nucleus, putamen) in PD/T2 and FLAIR and even better seen in dorsomedial thalamic nuclei, what gave appearance characteristic for Heidenhain variant of the CJD (so called "hockey-stick") (Figs. 2, 3A-C). Similar findings were noted in occipital lobe cortex (Fig. 3C).

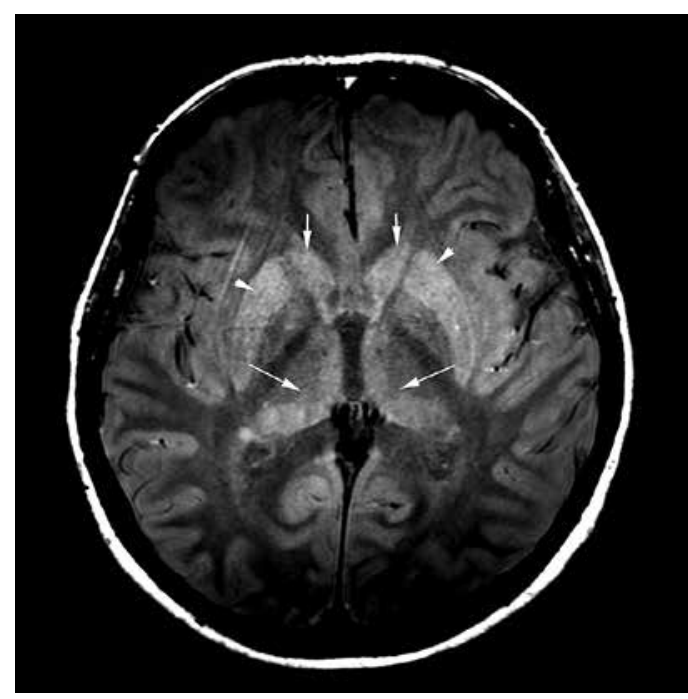

Fig. 2. Brain MRI, axial plane, PD/T2 sequence: Symmetrical hyperintensity of the basal ganglia: caudate nucleus (short arrow), putamen (arrow head) and in dorsomedial thalamic nucleus, "hockey-stick" appearance (long arrow), characteristic for Heidenhain variant of the CJD. 


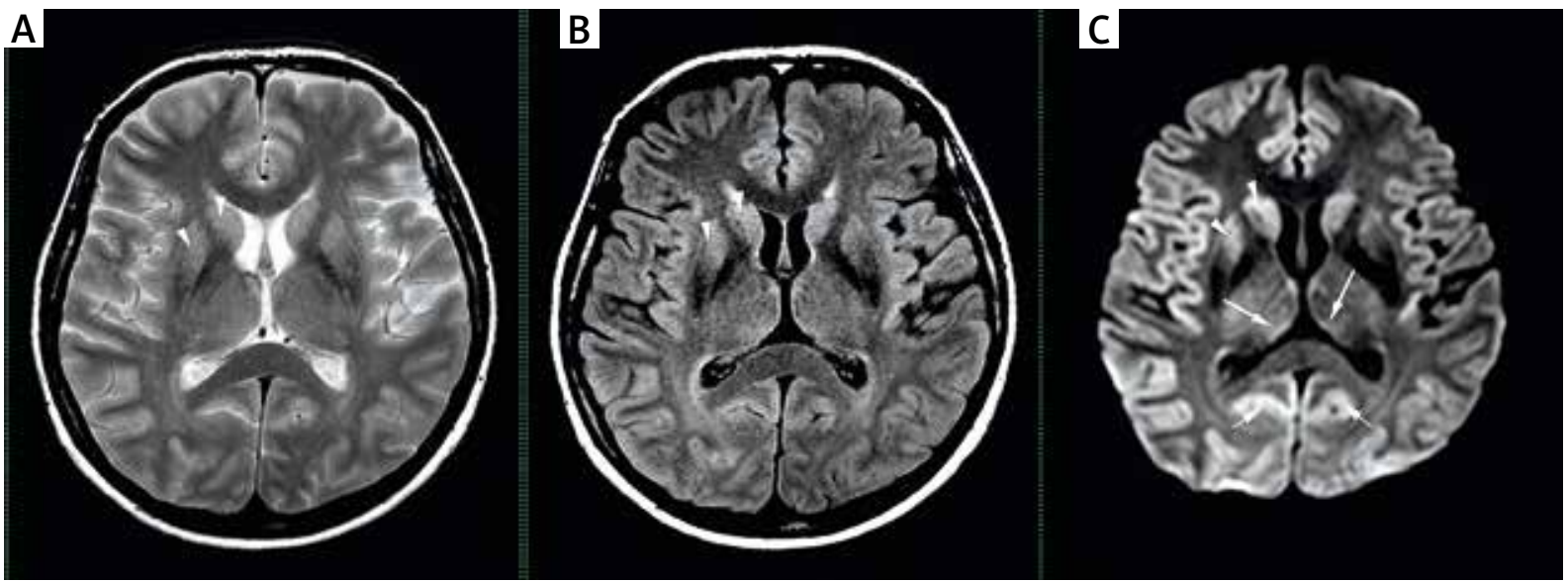

Fig. 3. Brain MRI, axial plane, sequences: A) TSET2, B) FLAIR T2, C) DWI SENSE: A, B) symmetrical hyperintensity of the basal ganglia in caudate nucleus, putamen (arrowheads), C) symmetrical hyperintensity of the basal ganglia in caudate nucleus, putamen (arrowheads), the occipital lobe cortex (short arrows) and - poorly seen only in DWI - dorsomedial thalamic nucleus (long arrows).

As a result, just before the death she fulfilled the NCJDSU criteria for probable CJD.

To definitely confirm the CJD the brain autopsy was performed in the Department of Neuropathology, Collegium Medicum Jagiellonian University (description given below). Moreover, the CSF sample (taken before the demise of the patient) was sent again for 14-3-3 protein analysis (with ELISA method) and RT-QulC test to the National Reference Centre for the surveillance transmissible spongiform encephalopathy in Gottingen (Germany). The autopsy and the aforementioned test turned out to be positive thus making the diagnosis of CJD definite.

The neuropathological examination revealed moderate and symmetrical general (cortical and subcortical) cerebral atrophy. The microscopic evaluation (Fig. 4A-C) disclosed spongiform changes in grey matter, diffused astrocytosis and microgliosis as well as neuronal loss in the brain hemispheres (neocortex, basal ganglia and thalamus). In the region of basal ganglia, thalamus, occipital and parietal cortex the so-called "synaptic-like" pattern of PrP accumulation was found. The "kuru-like" plaques were not identified. The distribution and extent of these neuropathological changes as well as the type of prion immunopositivity were suggestive of the sporadic variant of CJD, most probably with MM1 subtype of the prion.

\section{Discussion}

Creutzfeldt-Jakob disease is a very rare and rapidly progressive neurodegenerative disorder with the fatal outcome. It is caused by an abnormal form of the prion protein. The normal form of the prion
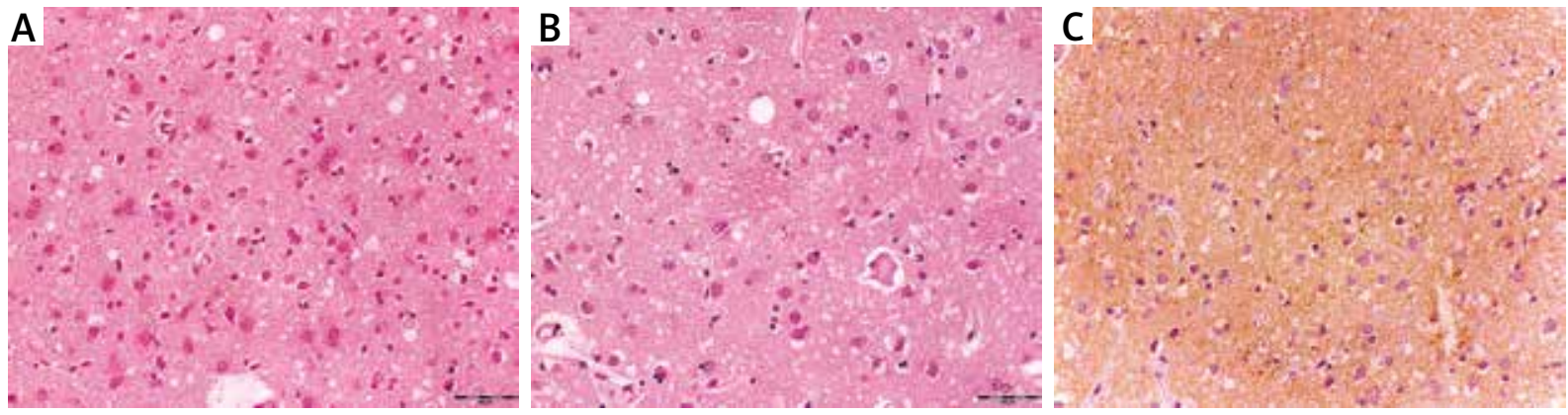

Fig. 4. A) Loss of neurons, severe astrogliosis and spongiform change in cerebral cortex. B) Spongiform change with mild loss of neurons in thalamus. C) Synaptic pattern of prion PrP immunoexpression (A, B - haematoxylin-eosin, C - PrP immunohistochemistry). 
protein $(\operatorname{PrPC})$, which is expressed at the highest level in neurones within the brain, is converted into an abnormal isoform (designated $\mathrm{PrPSC}$ ). The defective prion protein accumulates in the CNS and results in neurodegeneration.

The neuropathology of CJD consists of spongiform change, astrogliosis and microgliosis and neuronal loss [11]. The details of the misfolding site and its precise mechanism remain unclear [5]. CJD occurs in four types: sporadic (SCJD), familial or genetic (fCJD), iatrogenic (iCJD) and variant CJD (vCJD) [11].

The natural polymorphism of human prion protein gene (PRNP) at codon 129 which encodes either methionine $(M)$ or valine $(W)$ determins the susceptibility to sporadic and acquired forms of prion disease as well as its clinicopathological characteristics. On the basis of codon 129 polymorphism and physicochemical properties of type $1 \mathrm{PrPSC}$ or type $2 \mathrm{PrPSC}$, SCJD can be classified into six subtypes: MM1, MM2, MV1, MV2, VV1 and VV2 [6].

The clinical presentation as well as the molecular and neuropathological pictures reveal the heterogeneity of CJD phenotypes, however the dynamic dementia is its common denominator. The diagnosis of CJD faces a big challenge during the lifetime due to the overlapping clinical syndromes. The progressive decline in the neurological condition is followed by focal neurological signs with myoclonus being the most typical [14]. The commonly reported neurological abnormalities include the visual changes leading to cortical blindness, ataxia, pyramidal and extrapyramidal features and usually an akinetic mutism in the last stages of the disease [7]. The atypical findings such as behavioural abnormalities (anxiety, irritability, social withdrawal), subtle memory changes, judgment difficulties, and other psychiatric symptoms, are frequently observed in the early stages of the disease but can be easily overlooked [12].

The estimated incidence of human prion disease is about 1-2 persons per million worldwide annually [2]. The vast majority of human prion diseases are sporadic (SCJD) and account for $85 \%$ of CJD cases. The initial symptoms of SCJD occur in the $7^{\text {th }}$ decade of life and the median lifetime expectancy is 5 months with $90 \%$ of patients passing away within 1 year.

Approximately $10 \%$ of all CJD cases are familial/ genetic. The familial CJD is caused by diverse mutations in the PRNP gene. The disease is transmitted in autosomal dominant pattern with high penetrance and an incidence increasing with age. The inherited forms are classified into three categories: Gerstmann-Straussler-Scheinker syndrome, fatal familial insomnia (FFI) and fCJD [7].

The acquired form of CJD (including ICJD and vCJD) occurs in $2-5 \%$ of cases [12]. The form iCJD is a consequence of transmission of abnormal prion protein during medical procedures. Over the last few decades it was described after intracerebral electrode implantation, corneal transplantation, dura mater grafts, and growth hormone injections. latrogenic CJD's clinical picture as well as MRI and EEG findings are similar to SCJD [7].

Variant CJD was first described in 1996 and as a result of exposure (including ingestion) to the products of animals suffering from bovine spongiform encephalopathy (BSE). The clinical presentation, neuroimaging and pathological findings differ from other variants of CJD. The most cases of VCJD had presented psychiatric symptoms in the early course before the ataxia began approximately within 6 months. Its onset was reported in younger subjects than in SCJD or FCJD. The research showed that the median age of its onset was 27 years (range: 12-74) in the UK, 35 years in France (range: 18-57) and the median duration of the disease was 14 months for both countries [2]. The studies revealed the presence of the pathological form of prion protein in peripheral tissues of these patients (tonsil, lymph nodes, appendix, spleen), what was suggested to be used for diagnostic purpose [14].

The first diagnostic criteria for CJD were formulated by the World Health Organization (WHO) in 1998 (Table II) with the diagnosis relying on clinical examination, EEG, and CSF findings [7].

The present internationally recognised criteria published in 2017 by the National CJD Research and Surveillance Unit (NCJDRSU) in Edinburgh have been expanded to include brain MRI findings and modern laboratory tests. On the basis of NCJDRSU criteria, SCJD (Table III) can be qualified as define, probable or possible [9].

The EEG was found as the first significant and non-invasive test to point the diagnosis of CJD. Its importance has been emphasized and included in the first published diagnostic criteria [4]. The characteristic pattern of periodic sharp and slow wave complexes (PSWCS) was reported in two-thirds of cases of SCJD. The typical appearance for SCJD is that of a $1 /$ second periodic triphasic sharp wave 
Table II. The 1998 WHO diagnostic criteria for CJD

\begin{tabular}{|c|c|}
\hline \multicolumn{2}{|c|}{ Diagnostic criteria for CJD } \\
\hline A & Rapidly progressive dementia \\
\hline \multirow[t]{5}{*}{ B } & Specific neurological manifestations: \\
\hline & 1. Myoclonus \\
\hline & 2. Visual or cerebellar problems \\
\hline & 3. Pyramidal or extrapyramidal features \\
\hline & 4. Akinetic mutism \\
\hline \multirow[t]{3}{*}{ C } & Laboratory tests: \\
\hline & $\begin{array}{l}\text { 1. PSWCS on EEG during an illness of any } \\
\text { duration }\end{array}$ \\
\hline & 2. Positive CSF: 14-3-3 protein \\
\hline \multicolumn{2}{|l|}{ Classification } \\
\hline Definite sCJD & $\begin{array}{l}\text { Requires neuropathological/ } \\
\text { immunocytochemical confirmation }\end{array}$ \\
\hline Probable sCJD & $A+$ at least 2 of $B+$ at least 1 of $C$ \\
\hline Possible sCJD & $A+$ at least 2 of $B+$ duration $<2$ years \\
\hline
\end{tabular}

complex. The simple sharp waves can be classic triphasic, biphasic, or mixed [7].

This characteristic pattern in some patients was observed as early as three weeks after onset of the disease. However, in majority of cases it occurs about twelve weeks after onset and in a few isolated cases even later. In contrast to the familial and sporadic types, the EEG study is not usually informative in iatrogenic human growth hormone cases as well as it is negative for any periodic sharp wave forms in patients with VCJD. In our case, the EEG revealed high-voltage theta and delta waves on the right hemisphere with periodic epileptic discharges, however the pattern was not typical for CJD. Moreover, these epileptic discharges were observed in the region of suspected meningioma, thus could have potentially explained their presence. Later on, EEG showed non-specific slow waves abnormalities.

The results of CSF analysis in standard investigations (cell count, barrier function and inflammatory reactions) in patients with CJD are in general within normal range [14]. A slightly elevated protein (0.5-1.0 g/l) was noted in one-third of cases. The presence of oligoclonal bands in CSF was very rarely observed $[4,14]$.

Several studies reported the biomarkers that can be useful in diagnosis of CJD, including protein 14-3-3, tau, S100b, neuron-specific enolase and phosphorylated tau. The majority of available data relate to 14-3-3 and tau proteins [4]. The detection of 14-3-3 protein in the CSF by using a Western-Blot method
Table III. Clinical criteria for Creutzfeldt-Jakob disease (CJD) - NCJDRSU in Edinburgh 2017 [9]

\begin{tabular}{|c|c|}
\hline I & Progressive cognitive impairment \\
\hline \multirow[t]{4}{*}{ II } & A Myoclonus \\
\hline & B Visual or cerebellar problems \\
\hline & C Pyramidal or extrapyramidal features \\
\hline & D Akinetic mutism \\
\hline III & Typical EEG: PSWCS \\
\hline IV & $\begin{array}{l}\text { High signal in caudate/putamen on MRI brain } \\
\text { scan or at least two cortical regions (temporal, } \\
\text { parietal, occipital) either on DWI or FLAIR }\end{array}$ \\
\hline \multicolumn{2}{|c|}{ Definite } \\
\hline \multicolumn{2}{|c|}{$\begin{array}{l}\text { Progressive neurological syndrome AND at least } \\
\text { neuropathologically OR immunocytochemically } \\
\text { or biochemically confirmed }\end{array}$} \\
\hline \multicolumn{2}{|c|}{ Probable } \\
\hline \multicolumn{2}{|c|}{$I+$ at least 2 of $I I$ and $I I I$} \\
\hline \multicolumn{2}{|l|}{ or } \\
\hline \multicolumn{2}{|c|}{$I+$ at least 2 of II and IV } \\
\hline \multicolumn{2}{|l|}{ or } \\
\hline \multicolumn{2}{|c|}{$I+$ at least 2 of II and positive $14-3-3$} \\
\hline \multicolumn{2}{|l|}{ or } \\
\hline \multicolumn{2}{|c|}{$\begin{array}{l}\text { Progressive neurological syndrome and positive RT-QuIC } \\
\text { in CSF or other tissues }\end{array}$} \\
\hline \multicolumn{2}{|c|}{ Possible } \\
\hline \multicolumn{2}{|c|}{$I+$ at least 2 of $I I+$ duration $<2$ years } \\
\hline
\end{tabular}

occurred to be sensitive and specific. The protein $14-3-3$ is a neuronal destruction marker and can be present in early stages of the clinical disease [4]. Its sensitivity is estimated at $92-96 \%$ in SCJD, what is in contrast to lower sensitivity of 50\% in VCJD [14]. The protein 14-3-3 in fCJD patients bearing codon 200 and codon 210 mutations has a similar diagnostic value as in SCJD. In case of the fatal familial insomnia the protein 14-3-3 is constantly absent in the CSF as well as it is uncommon in GSS. The sensitivity is also low in patients with iCJD (60\%) but may increase in later stages of the disease [14].

The neurological conditions that cause neuronal loss may also give positive results of 14-3-3 protein in CSF, but their clinical presentation should be differentiated from CJD. The presence of the 14-3-3 protein in CSF was found in the following diseases: herpes simplex and other viral encephalitis, recent stroke, subarachnoid haemorrhage, hypoxic brain damage, metabolic encephalopathy after barbiturate intoxication, glioblastoma, carcinomatous meningitis from small-cell lung cancer, paraneoplastic encephalopathy, and corticobasal degeneration [4]. 
The RT-QuIC assays of CSF is a modern laboratory technique that enables definitive diagnosis of CJD. It provides detection of an abnormal form of prion protein $\left(\mathrm{PrP}^{\mathrm{SC}}\right)$ through in vitro amplification technology in CSF samples $[1,7]$. The test has sensitivity estimated at $80-90 \%$, but in contrast to the test for 14-3-3 protein, its specificity is $100 \%$. The difference in detection among the genetic subtypes was not reported [7].

The conducted studies demonstrated a significant role of $\mathrm{MRI}$ in the diagnostic process of CJD. The cerebral cortical hyperintensities as well as the high signal in caudate nucleus and putamen on fluid attenuated inversion recovery (FLAIR) or diffusion-weighted imaging (DWI) MRI have been reported as the characteristic lesion features of sporadic Creutzfeldt-Jakob disease [13]. The typical signal enhancements in VCJD were reported in the posterior thalamus and termed "pulvinar sign" [14]. The key is the knowledge of the disease state while interpreting neuroimaging. It is still unclear when changes in the typical findings appear. However, there are some suggestions that characteristic MRI patterns may be presented in early stages [13]. DWI was found to be superior to any other MRI sequence in early stages of CJD. It was reported that in the course of the condition the hyperintensity decreases in later stages and then the cortical atrophy is observed [14]. In relation to our patient the MRI turned out to be characteristic for CJD in the late phase of the disease. The first brain MRI was performed two months before admission to the ward and apart from the suspected meningioma in the right fronto-parietal region, there were no other abnormalities. During the hospitalisation she underwent two brain MRI. The initial one was difficult to analyse due to motor artifacts, but some hyperintensity in the region of the right caudate and the inner capsule on DWI was observed. The second MRI, performed two weeks later, revealed lesions characteristic for Heidenhain variant of the CJD.

The definite diagnosis of SCJD requires neuropathological examination. The common histopathological changes confined to the CNS include: spongiform vacuolation throughout the cerebral grey matter, reactive proliferation of astrocytes and microglia, neuronal loss, and PrP deposition within the brain. However, the mentioned features are not specific for the prion disease. Their occurrence in defined neuroanatomical regions of the brain is of significant importance in the differential diagnosis of the disease [6].

In our case the diagnosis of SCJD was considered during the early course of the disease, but the initial results of the conducted examinations were not diagnostic. The first brain MRI was difficult to analyse due to a lot of motor artefacts, however, the follow-up neuroimaging revealed the characteristic lesions for CJD. The EEG was not specific for CJD. We obtained the negative results of 14-3-3 protein in the first CSF analysis, but the follow-up one was of significant importance and confirmed the diagnosis together with a more sensitive RT-QulC test.

We were speculating on the reason for the discrepancy in the results of both received tests detecting 14-3-3 protein. Both of the tests vary in the used method, localisation of the laboratory and two different time points of testing. The second sample was tested with the use of ELISA technique instead of Western-Blot in the first test. Both of the mentioned techniques are most commonly used to detect 14-3-3 protein. One of conducted studies comparing ELISA technique with Western-Blot in detecting 14-3-3 protein showed that, after combining definite and probable cases as a reference, the sensitivities were 88.9\% for ELISA and $93.7 \%$ for Western-Blot, with a specificity of $97.6 \%$ for both methods. The authors of mentioned studies argued that ELISA might give more consistent outcomes that are less likely to confound the assessment seen in the Western-Blot method. On the other hand, another study conducted on 253 patients revealed no significant difference in specificity and sensitivity between the Western-Blot technique and the ELISA technique [8]. More recent data indicate that both of the methods give comparable results and their authors draw attention to quality of the analysis in particular the standardization of the preanalytical treatment of CSF samples [10].

The next research performed on 32 cases with pathologically confirmed SCJD displayed sensitivity of only $53 \%$ for testing $14-3-3$ protein in both techniques and found the significant relationship between positive 14-3-3 results and shorter time from disease onset to the test, what is consistent with the argument that 14-3-3 protein is associated with acute neuronal loss (the patients with rapid progress of the disease are more likely to undergo lumbar puncture and 14-3-3 protein test earlier than those with slower illness) [3]. 
Thus, in the context of our case, we reflect on whether gradual progression of neurological symptoms during first months of the disease (period before hospitalization) could explain the lack of 14-3-3 protein in CSF in the initial examination (as well as lack of abnormalities in the brain MRI two months before admission). The fulminant deterioration of her condition observed in the ward resulted in acute neuronal loss and then the positive result of 14-3-3 protein presence in the control test was obtained.

The researchers suggest that in doubtful cases, the serial testing of CSF should be considered due to an observed increase in sensitivity of 14-3-3 protein investigation in later stages of SCJD. Therefore, it is recommended to repeat lumbar puncture at least within 2 weeks after the initial one as was in our case [14].

\section{Conclusions}

The confirmation of CJD is a diagnostic challenge that demands an extensive differential diagnosis and a series of conducted tests should be considered especially in cases with suspected "false-negative" results in initial examinations. The clinical symptoms can be masqueraded by other neurological syndromes, as was in our case during the long prehospitalisation period of the disease. There is a need for standardisation of 14-3-3 protein analysis. Currently the most specific test for CJD is RT-QuIC, but the "the gold standard" of making definite diagnosis is neuropathological examination.

\section{Acknowledgments}

We thank the members from the National TSE Reference Centre in Gottingen, Germany for kindly providing the necessary investigations to confirm the diagnosis.

\section{Disclosure}

The authors report no conflict of interest.

\section{References}

1. Atarashi R, Sano K, Satoh K, Nishida N. Real-time quakinginduced conversion: a highly sensitive assay for prion detection. Prion 2011; 5: 150-153.

2. Chen C, Dong X. Epidemiological characteristics of human prion diseases. Infect Dis Poverty 2016; 5: 47.
3. Geschwind MD, Martindale J, Miller D, DeArmond SJ, Uyehara-Lock J, Gaskin D, Kramer JH, Barbaro NM, Miller BL. Challenging the clinical utility of the 14-3-3 protein for the diagnosis of sporadic Creutzfeldt-Jakob disease. Arch Neurol 2003; 60: 813816.

4. Global surveillance, diagnosis and therapy of human transmissible spongiform encephalopathies: report of a WHO consultation, Geneva, Switzerland, 9-11 February 1998. https://apps. who.int/iris/handle/10665/65516.

5. Ironside J. Variant Creutzfeldt-Jakob disease: an update. Folia Neuropathol 2012; 50: 50-56.

6. Iwasaki Y. Creutzfeldt Jakob disease. Neuropathology 2017; 37: 174-188.

7. Manix M, Kalakoti P, Henry M, Thakur J, Menger R, Guthikonda B, Nanda A. Creutzfeldt-Jakob disease: updated diagnostic criteria, treatment algorithm, and the utility of brain biopsy. Neurosurg Focus 2015; 39: E2.

8. Muayqil, Taim \& Gronseth, Gary \& Camicioli, Richard. (2012). Evidence-based guideline: diagnostic accuracy of CSF 14-33 protein in sporadic Creutzfeldt-Jakob disease: report of the guideline development subcommittee of the American Academy of Neurology. Neurology 2012; 79: 1499-1506.

9. National CJD Research \& Surveillance Unit, University of Edinburgh, Protocol Surveillance of CJD in the UK, 2017 April. https://www.cjd.ed.ac.uk/sites/default/files/criteria.pdf.

10. Schmitz M, EbertE, Stoeck K, Karch A, Collins S, Calero M, Sklaviadis T, Laplanche JL, Golanska E, Baldeiras I, Satoh K, Sanchez-Valle R, Ladogana A, Skinningsrud A, Hammarin AL, Mitrova E, Llorens F, Sun Kim Y, Green A, Zerr I. Validation of 14-3-3 protein as a marker in sporadic Creutzfeldt-Jakob disease diagnostic. Mol Neurobiol 2016; 53: 2189-2199.

11. Sikorska B, Knight R, Ironside JW, Liberski PP. Creutzfeldt-Jakob disease. In: Ahmad SI (Ed.). Neurodegenerative diseases. Advances in experimental medicine and biology. Vol. 724 . Springer, New York, NY 2012.

12. Tan B, Morales Mangual C, Mahmud I, Tongo ND, Mararenko L, Kay A. A case report of probable sporadic Creutzfeldt-Jakob disease: how to approach early diagnosis? Cureus 2017; 9: e1297.

13. Zerr I, Kallenberg K, Summers DM, Romero C, Taratuto A, Heinemann U, Breithaupt M, Varges D, Meissner B, Ladogana A, Schuur M, Haik S, Collins S, Jansen GH, Stokin GB, Pimentel J, Hewer E, Collie D, Smith P, Roberts H, Brandel JP, van Duijn C, Pocchiari M, Begue C, Cras P, Will RG, Sanchez-Juan P. Updated clinical diagnostic criteria for sporadic Creutzfeldt-Jakob disease. Brain 2009; 132 (Pt 10): 2659-2668. Erratum in: Brain 2012; 135 (Pt 4): 1335.

14. Zerr I, Poser S. Clinical diagnosis and differential diagnosis of CJD and VCJD. With special emphasis on laboratory tests. APMIS 2002; 110: 88-98. 